TecnoLógicas

ISSN-p 0123-7799

ISSN-e 2256-5337

Vol. 22, No. 45 , pp. $145-153$

Mayo-agosto de 2019

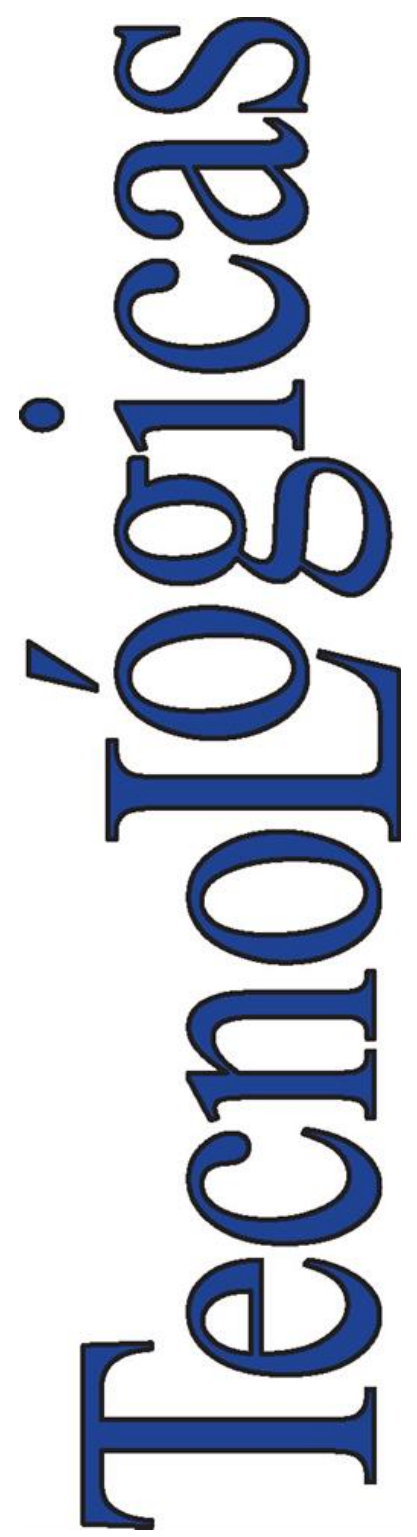

(C) Instituto Tecnológico Metropolitano Este trabajo está licenciado bajo una Licencia Internacional Creative

Commons Atribución (CC BY-NC-SA)

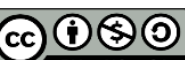

Artículo de Investigación/Research Article

\section{Cambios en los compuestos bioactivos del jugo de uchuva (Physalis peruviana L.) pasteurizado}

\author{
Changes in the bioactive compounds of \\ pasteurized gooseberry (Physalis peruviana L.) \\ juice
}

\author{
Lilian Cárcamo-Medina (D) ${ }^{1}$, Mildred Elezar-Turcios(D22, \\ y Luis Eduardo Ordóñez-Santos (D) ${ }^{3}$
}

Recibido: 19 de marzo de 2019

Aceptado: 30 de abril de 2019

Cómo citar / How to cite

L. Cárcamo-Medina, M. Elezar-Turcios, y L. E. Ordóñez-Santos, "Cambios en los compuestos bioactivos del jugo de uchuva (Physalis peruviana L.) Pasteurizado," TecnoLógicas, vol. 22, no. 45, pp. 145-153, 2019. https://doi.org/10.22430/22565337.1279

1 Estudiante de Tecnología Alimentaria, Facultad de Ciencias Tecnológicas, Universidad Nacional de Agricultura, Catacamas-Honduras, lcarcamo@unal.edu.co

2 MSc en Tecnología de Alimentos, Facultad de Ciencias Tecnológicas, Universidad Nacional de Agricultura, Catacamas-Honduras, eleturcios@unag.edu.hn

$3 \mathrm{PhD}$ en Ciencias Biológicas, Facultad de Ingeniería y Administración, Universidad Nacional de Colombia, Palmira-Colombia, leordonezs@unal.edu.co 


\section{Resumen}

El mercado mundial de bebidas de frutas está interesado en jugos exóticos con alto valor nutricional y parte de esa demanda puede satisfacerse con derivados de uchuva. Por lo tanto, el objetivo de este estudio fue evaluar el efecto de la pasteurización sobre la concentración de vitamina $\mathrm{C}$, carotenoides y el color superficial del jugo de uchuva. Frutos de uchuva en estado de madurez 5 (amarillo-naranja) se procesaron en un extractor de jugo comercial y el jugo obtenido se mezcló con agua destilada en una relación 1:1. Se evaluaron dos tratamientos de pasteurización $\left(80{ }^{\circ} \mathrm{C}\right.$ durante 15 minutos y $94{ }^{\circ} \mathrm{C}$ durante 29 minutos) para conocer sus efectos sobre las variables de respuesta (vitamina $\mathrm{C}$, carotenoides y color superficial). Los resultados indican que los tratamientos de pasteurización no afectaron significativamente la concentración de vitamina $\mathrm{C}$ y la luminosidad. Sin embargo, los tratamientos térmicos aumentaron significativamente $(\mathrm{p}<0,05)$ entre 50 a $83 \%$ la concentración de carotenoides, y los valores de cromaticidad e índice de amarillamiento en el jugo de uchuva. De acuerdo con los resultados, se puede concluir que los tratamientos de pasteurización favorecen la calidad nutricional y el color superficial del jugo de uchuva.

\section{Palabras clave}

Carotenoides, vitamina $\mathrm{C}$, fruta exótica, luminosidad, cromaticidad.

\section{Abstract}

The worldwide market of fruit drinks is interested in exotic juices with high nutritional value, and part of that demand can be satisfied with gooseberry derivatives. Therefore, the objective of this study was to evaluate the effect of pasteurization on the concentration of vitamin $\mathrm{C}$, carotenoids, and surface color of cape gooseberry juice. Gooseberry fruits in a state 5 of maturity (yellow-orange) were processed in a commercial juice extractor, and the obtained juice was mixed with distilled water in a 1: 1 ratio. Two pasteurization treatments $\left(80^{\circ} \mathrm{C}\right.$ for 15 minutes and $94^{\circ} \mathrm{C}$ for 29 minutes) were evaluated to establish their effects on the response variables (vitamin $\mathrm{C}$, carotenoids, and surface color). The results indicate that the pasteurization treatments did not significantly affect the concentration of vitamin $\mathrm{C}$ or the luminosity. Nevertheless, the thermal treatments significantly increased $(\mathrm{p}<0.05)$, between 50 and $83 \%$, the concentration of carotenoids, the values of chromaticity, and the yellowing index of cape gooseberry juice. According to the results, it can be concluded that pasteurization treatments favor the nutritional quality and superficial color of said juice.

\section{Keywords}

Carotenoids, vitamin C, exotic fruit, luminosity, chromaticity. 


\section{INTRODUCCIÓN}

En 2015, se consumieron 38,473 millones de litros de jugo de fruta en todo el mundo [1]. El mercado mundial de jugos de frutas está interesado en sabores exóticos y con alto contenido de micronutrientes [2]. La uchuva es una fruta que tiene gran importancia en Colombia, por ello, se ha convertido en la fruta exótica de mayor exportación en el país [3], encontrándose en ella una sustancial fuente de compuestos bioactivos, como el ácido ascórbico, vitamina $\mathrm{A}$, complejos de vitamina $\mathrm{B}$, minerales, $\mathrm{y}$ carotenoides [4]. Estos compuestos están asociados a la prevención de múltiples enfermedades, ya que actúan como antiinflamatorios, anticancerígenos, antidiabéticos y previenen enfermedades cardiovasculares y coronarias [3], [5].

Durante el proceso de elaboración de jugo de frutas, la inactivación de microorganismos y enzimas mediante el tratamiento térmico de pasteurización, permite prolongar la vida útil, al conservar la calidad nutricional y sensorial de este tipo de producto [6], [7]. El efecto de la pasteurización en la calidad del jugo de frutas ha sido abordado en diferentes investigaciones, por ejemplo, en jugo de piña [8], jugo de acerola [9], jugo de naranja [10] y jugo de uchuva [2], [11].

A pesar del potencial agroindustrial de esta fruta, no existe en la literatura científica, suficientes estudios que informen de los cambios en los compuestos bioactivos después de la pasteurización del jugo de uchuva elaborado con frutos cultivados en Colombia. Por lo tanto, esta investigación tuvo como propósito evaluar el efecto de la pasteurización sobre la concentración de vitamina $\mathrm{C}$, carotenoides y color superficial del jugo de uchuva.

\section{METODOLOGíA}

\subsection{Localización}

El trabajo de investigación se realizó en el laboratorio de Tecnología de Frutas y Hortalizas de la Universidad Nacional de Colombia, sede Palmira, localizada a 1001 m.s.n.m. y una temperatura promedio de $23{ }^{\circ} \mathrm{C}$.

\subsection{Materia prima}

Se utilizaron frutos de uchuva en estado de madurez 5 (amarillo-naranja) según la tabla de color NTC 4580 [12] cultivadas en el municipio de Silvia, Cauca a 2800 m.s.n.m. Los frutos fueron trasladados al laboratorio de Tecnología de Frutas y Hortalizas de la Universidad Nacional de Colombia donde se almacenaron por $24 \mathrm{~h}$ a $2{ }^{\circ} \mathrm{C}$.

\subsection{Preparación de jugo}

Los frutos fueron seleccionados según el estado de madurez (amarillo-naranja) y por ausencia de daños mecánicos y microbiológicos, luego se lavaron solo con agua, se llevaron los frutos enteros a un extractor comercial de jugo donde se extrajo el jugo eliminando las semillas o partículas gruesas. El jugo se diluyó en agua destilada (relación 1: 1) de acuerdo con [13]. Posteriormente, se cubrió con papel aluminio y se almacenó por $24 \mathrm{~h}$ a 2 ${ }^{\circ} \mathrm{C}$ antes de los tratamientos térmicos.

\subsection{Tratamientos térmicos}

El procesado térmico en el jugo de uchuva se llevó a cabo de acuerdo con [14], ya que se garantiza un calentamiento isotérmico en las muestras de estudio. Se depositaron $10 \mathrm{ml}$ de jugo en cada tubo de ensayo de vidrio. Los tubos fueron previamente envueltos con papel aluminio para evitar cualquier interferencia de luz. Luego se procedió a realizar la 
pasteurización en un baño de agua con cabina cerrada para evitar la presencia de oxígeno durante el procesado térmico. Los tratamientos de pasteurización evaluados fueron $\mathrm{TT} 1=80{ }^{\circ} \mathrm{C}, 15 \mathrm{~min}, \mathrm{y} \mathrm{TT} 2=94{ }^{\circ} \mathrm{C}$, 29 min, todos los tratamientos se realizaron por triplicado. El TT1 se utilizó sobre la base de reducir al menos 5 órdenes logarítmicas en el recuento microbiano definido por norma estándar internacional para jugos de frutas Food and Drug Administration FDA [15], y el TT2 se escogió con el fin de evaluar el comportamiento del tratamiento con mayor temperatura y mayor tiempo de exposición al calor. Las muestras se enfriaron inmediatamente a $25{ }^{\circ} \mathrm{C}$, sumergiéndolas en un vaso de precipitación de vidrio con agua fría a $2{ }^{\circ} \mathrm{C}$ durante 1 min y después se almacenaron por $24 \mathrm{~h}$ a $2^{\circ} \mathrm{C}$ hasta un análisis posterior [2].

\subsection{Análisis fisicoquímico}

El pH, los sólidos solubles totales (SST) y la acidez (\% de ácido cítrico) se realizaron de acuerdo con [16]. El pH del jugo de uchuva se midió en un potenciómetro (Mettler FE20, Mettler Toledo, Shanghai, China). Los sólidos solubles totales se estimaron a $20{ }^{\circ} \mathrm{C}$ como ${ }^{\circ}$ Brix con un refractómetro Abbe (WYA-2 W, Shanghai Precision y Scientific Instrument Co. Ltd., China).

\subsection{Medición del color}

El color se midió de acuerdo con [2], usando un colorímetro Kónica Minolta CR400, en coordenadas CIEL*a*b* $\left(L^{*}, a^{*} y\right.$ $\left.\mathrm{b}^{*}\right)$; usando un iluminante D65 y un observador de $2^{\circ}$, con parámetros de calibración de $\mathrm{Y}=89,5 ; \mathrm{x}=0,3176 ; \mathrm{y}=0,3347$. Los valores de cromaticidad $\left(\mathrm{C}^{*}\right)$, índice de amarillo $\left(\mathrm{IA}^{*}\right)$ y tono $\left(\mathrm{h}^{\circ}\right)$ se calcularon mediante las ecuaciones (1), (2) y (3), de acuerdo con [2]:

$$
\mathrm{C}=\left(a *^{2}+b *^{2}\right) 1 / 2
$$

$$
\begin{gathered}
I A=(142,86 b *) /(L *) \\
h=\tan ^{-1}(b * / a *)
\end{gathered}
$$

\subsection{Medición de la concentración del ácido ascórbico}

El contenido de ácido ascórbico se midió mediante el método de Folin-Ciocalteu [17]. Se diluyó $0,2 \mathrm{ml}$ de la muestra en 0,8 $\mathrm{ml}$ de ácido tricloroacético. Se refrigeró durante 5 min y luego se centrifugó a 3000 $\mathrm{rpm}$ por $5 \mathrm{~min}$. Se tomó $0,5 \mathrm{ml}$ del sobrenadante y se le agregó $2 \mathrm{ml}$ de agua biodestilada y $0,2 \mathrm{ml}$ del reactivo FolinCiocalteu. Se dejó en oscuridad durante 10 min para posteriormente medir la absorbancia en un espectrofotómetro a 760 $\mathrm{nm}$. La concentración de vitamina C (mg de ácido ascórbico / $100 \mathrm{ml}$ de jugo) en las muestras se calculó a partir de una recta de calibración preparada con una solución estándar de ácido ascórbico (0,0000125 a 0,000125 g /ml, R2 = 0,999).

\subsection{Determinación de la concentración de carotenoides}

Los carotenoides se extrajeron según [2]. Se pesó en un tubo $0,1 \mathrm{~g}$ de la muestra, luego se añadió $7 \mathrm{ml}$ de etanol/hexano (relación 4: 3); se colocaron los tubos en un vaso de precipitación de vidrio con agua fría cubierto por completo con papel aluminio. Se agitó por $1 \mathrm{~h}$ a $140 \mathrm{rpm}$, después se le adicionó $1 \mathrm{ml}$ de agua destilada a cada tubo y se continuó agitando por $10 \mathrm{~min}$. Se extrajo el sobrenadante (fase orgánica) y se procedió a leer a 450, 444, 451 y $472 \mathrm{~nm}$ respectivamente en un espectrofotómetro (espectrofotómetro Jenway 6320 D, England). La concentración de carotenoides $\mathrm{mg} / \mathrm{g}$ se calculó usando los coeficientes de extinción $(\mathrm{E} \% 1 \mathrm{~cm})$ en hexano: 2560, 2800, 2480 y 3540 para Bcaroteno, a-caroteno, B-criptoxantina, zeaxantina y licopeno respectivamente de acuerdo con [18]. 


\subsection{Diseño experimental}

El efecto de los tratamientos térmicos sobre las variables de respuesta, se evaluó con un diseño aleatorizado simple, con tres tratamientos (fresco $=$ jugo $\sin$ pasteurizar), $\left.\mathrm{TT} 1=80{ }^{\circ} \mathrm{C}, 15 \mathrm{~min}, \mathrm{y} \mathrm{TT} 2=94{ }^{\circ} \mathrm{C}, 29\right)$, con tres repeticiones por tratamiento. Se realizó un análisis de varianza de ANOVA con un nivel de significancia del $95 \%$ $(\mathrm{P}<0,05)$ y prueba de Tukey. Los análisis estadísticos se analizaron utilizando el programa SPSS 25 para Windows.

\section{RESULTADOS Y DISCUSIÓN}

En este estudio la pasteurización no afectó significativamente el $\mathrm{pH}$, la acidez y la vitamina $\mathrm{C}$ en el jugo de uchuva, mientras que los sólidos solubles y los carotenoides sí presentaron cambios significativos después del procesado térmico (Tabla 1). Resultados similares en pH y acidez han sido reportados en jugo uchuva y mango [2], [15]. En el caso de la vitamina $\mathrm{C}$ los resultados del presente estudio no se ajustan a los reportados por [2], [11] en jugo de uchuva, quienes informan una reducción de este antioxidante después de los tratamientos térmicos, mientras que en la investigación de [19], llegaron a la conclusión de que esta fruta puede ser hasta 15 veces más estable al calor que otras fuentes de vitamina $\mathrm{C}$, ya que la uchuva presentó concentraciones relevantes de ácido ascórbico aun después de ser tratado térmicamente. Una explicación del nivel de retención de la vitamina $\mathrm{C}$ en el jugo de uchuva pasteurizado, se encuentra en la posible inactivación de la enzima ascorbatasa, la remoción del oxígeno residual, y la protección a la fotodegradación durante el procesado térmico [20]. La variación de la concentración de vitamina $\mathrm{C}$ frente a otras investigaciones se dio posiblemente a los diferentes procedimientos en la elaboración del jugo, por ejemplo, en el presente estudio las uchuvas no se cortaron ni pelaron y estudios afirman que estos procesos provocan grandes pérdidas de vitamina $\mathrm{C}$ por efecto de la luz, oxígeno, temperatura, presión, azúcares reductores, pH y los iones metálicos [21]. De hecho, con solo realizar el cortado y pelado de las frutas y hortalizas (sin necesidad de haber aplicado calor), se da una pérdida importante de vitamina C [22]. Además, es importante recalcar que el proceso de extracción del jugo se hizo en un tiempo corto, teniendo control con la luz (forrando con papel aluminio todos los materiales utilizados), y se realizó el tratamiento térmico en una cabina de baño maría cerrada para evitar presencia de oxígeno, que es la principal causa de destrucción de vitamina $\mathrm{C}$ [23].

Los sólidos solubles se redujeron significativamente en el jugo de uchuva después del proceso de pasteurización (Tabla 1). Esta reducción probablemente se debe a procesos de lixiviación durante el procesado térmico del jugo de uchuva, que permitieron la extracción del agua presente en las vacuolas de las células vegetales. Estos resultados concuerdan con los obtenidos en jugos pasteurizados $\left(80^{\circ} \mathrm{C}\right.$ por $10 \mathrm{~min}$ ) de naranja, lima dulce, zanahoria y espinaca [24]; mientras que en jugo de fresa no se presentó diferencia en los sólidos solubles tratado a $85^{\circ} \mathrm{C}$ durante $2 \min [25]$.

Los tratamientos de pasteurización en el jugo de uchuva, aumentaron significativamente la concentración de todos los carotenoides frente al control (Tabla 1). El aumento en la concentración de carotenoides frente al control, difieren a los registrados en otros estudios, es así que, en jugo de melocotón, el B-caroteno y la B-criptoxantina disminuyeron después de ser sometido a pasteurización a $90{ }^{\circ} \mathrm{C}$ durante 5 minutos [26]. En otro trabajo, en jugo de naranja valencia brasileña, se reportó una pérdida de $13 \%$ del a-caroteno después de ser tratado térmicamente a 95 ${ }^{\circ} \mathrm{C}$ por $2 \mathrm{~min}$ [27]. El aumento de los 
carotenoides en este estudio, se debe posiblemente a la presencia de piel de la fruta en el experimento, ya que, para realizar el jugo, la fruta solo se trituró.

Esto quiere decir que parte del jugo contenía partículas de piel y estas funciona como un reservorio. La piel es una fuente valiosa en carotenoides, por lo tanto, al entrar en contacto con el calor, esta libera los carotenoides ligados a ella, algo similar se encontró en los estudios de pulpa de tomate [28] y de pasta de tomate, donde los productos procesados con piel presentaron mayor concentración de carotenoides que los productos que estaban libres de piel [29]. Otra razón, es que estos pigmentos están ligados con proteínas por enlaces covalentes, pero al aplicar calor, se produce una hidrólisis que rompe la estructura (proteína-carotenoide) y esto permite que haya mayor liberación de los carotenoides [30], [31]. También es importante mencionar que en los alimentos tratados térmicamente, los carotenoides pueden ser extraídos con mayor facilidad y esto hace que sean más disponibles biológicamente [32].

Tabla 1. Efecto de tratamientos térmicos sobre características fisicoquímicas y compuestos bioactivos del jugo de uchuva. Fuente: autores.

\begin{tabular}{|c|c|c|c|c|c|c|c|c|c|}
\hline Tratamientos & $\mathrm{pH}$ & $\mathrm{SST}$ & Acidez & Vitamina $\mathrm{C}$ & $\begin{array}{c}\text { B- } \\
\text { caroteno }\end{array}$ & a-caroteno & $\begin{array}{c}\text { B- } \\
\text { criptoxantin } \\
\mathrm{a} \\
\end{array}$ & $\begin{array}{c}\text { Zeaxa } \\
\text { ntina }\end{array}$ & $\begin{array}{c}\text { Licopen } \\
\text { o }\end{array}$ \\
\hline \multirow[t]{2}{*}{ Fresco } & & $\begin{array}{c}7,57 \\
\pm\end{array}$ & & & & $4,44 \pm$ & $4,14 \pm$ & $4,11 \pm$ & $2,22 \pm$ \\
\hline & 0,01 & $0,15^{\mathrm{a}}$ & 0,00 & 0,73 & $0,18^{c}$ & $0,19^{c}$ & $0,12^{\mathrm{c}}$ & $0,12^{\mathrm{c}}$ & $0,04^{\mathrm{c}}$ \\
\hline \multirow[t]{2}{*}{ TT1 } & $3,61 \pm$ & $\begin{array}{c}7,06 \\
\pm\end{array}$ & $0,12 \pm$ & $35,18 \pm$ & $7,50 \pm$ & $6,69 \pm$ & $7,58 \pm$ & $7,52 \pm$ & $3,75 \pm$ \\
\hline & 0,00 & $0,13^{\mathrm{b}}$ & 0,01 & 1,37 & $0,16^{\mathrm{a}}$ & $0,38^{\mathrm{a}}$ & $0,27^{\mathrm{a}}$ & $0,27^{\mathrm{a}}$ & $0,22^{\mathrm{a}}$ \\
\hline TT2 & $\begin{array}{c}3,60 \pm \\
0,00\end{array}$ & $\begin{array}{c}7,03 \\
\pm \\
0,15^{\mathrm{b}}\end{array}$ & $\begin{array}{c}0,12 \pm \\
0,00\end{array}$ & $\begin{array}{c}37,97 \pm \\
1,42\end{array}$ & $\begin{array}{c}6,09 \pm \\
0,26^{b}\end{array}$ & $\begin{array}{c}5,23 \pm \\
0,23^{\mathrm{b}}\end{array}$ & $\begin{array}{c}5,64 \pm \\
0,24^{\mathrm{b}}\end{array}$ & $\begin{array}{c}5,59 \pm \\
0,24^{\mathrm{b}}\end{array}$ & $\begin{array}{c}2,63 \pm \\
0,20^{\mathrm{b}}\end{array}$ \\
\hline ANOVA & NS & $* * *$ & NS & NS & $* * *$ & $* * *$ & $* * *$ & $* * *$ & $* * *$ \\
\hline
\end{tabular}

Nota: Los valores con letras diferentes en la misma columna son significativamente diferentes ( $p<0,5)$ entre sí. Fresco (sin tratamiento de pasteurización). TT1:80 ${ }^{\circ} \mathrm{C}, 15 \mathrm{~min}$. TT2: $94{ }^{\circ} \mathrm{C}, 29 \mathrm{~min}$. SST: Sólidos Solubles Totales $\left({ }^{\circ} \mathrm{Bx}\right)$, Acidez (\% de ácido cítrico), Vitamina $\mathrm{C}(\mathrm{mg} / 100 \mathrm{ml}$ de jugo), Carotenoides (mg/g de jugo).

Tabla 2. Efecto de tratamientos térmicos sobre el color del jugo de uchuva. Fuente: autores.

\begin{tabular}{lcccc}
\hline Tratamientos & L & C & IA & h \\
\hline Fresco & $30,12 \pm 0,34$ & $10,02 \pm 0,07^{\mathrm{b}}$ & $45,27 \pm 0,89^{\mathrm{b}}$ & $107,74 \pm 0,29^{\mathrm{a}}$ \\
TT1 & $30,77 \pm 0,52$ & $11,40 \pm 0,24^{\mathrm{a}}$ & $50,88 \pm 0,66^{\mathrm{a}}$ & $105,87 \pm 0,57^{\mathrm{b}}$ \\
TT2 & $30,75 \pm 0,57$ & $11,80 \pm 0,20^{\mathrm{a}}$ & $53,11 \pm 1,66^{\mathrm{a}}$ & $104,34 \pm 0,47^{\mathrm{c}}$ \\
ANOVA & NS & $* * *$ & $* * *$ & $* * *$ \\
\hline
\end{tabular}


El análisis de ANOVA evidencia que los tratamientos térmicos no afectaron significativamente los valores de luminosidad $\mathrm{L}^{*}$, mientras que el procesado de calor aumentaron significativamente los atributos de color $\mathrm{C}^{*}$ e IA; por el contrario, $\mathrm{h}^{\mathrm{o}}$ se redujo significativamente (Tabla 2). Los cambios no significativos de los valores de luminosidad $\left(\mathrm{L}^{*}\right)$ durante los procesos de pasteurización pueden estar asociados a la ausencia de reacciones de Maillard. Resultados que concuerdan con los reportados por [2] en jugo de uchuva pasteurizado.

Los carotenoides están estrechamente relacionados con el color. Al aumentar la concentración de carotenoides en el jugo pasteurizado (Tabla 1), aumenta los valores de $C^{*}$ e IA, mientras que $h^{\circ}$ disminuye lo cual explica la mayor percepción de color amarillo en las muestras estudiadas (Tabla 2).

\section{CONCLUSIONES}

Los tratamientos de pasteurización favorecen la calidad nutricional y sensorial del jugo de uchuva, ya que logran conservar el contenido de vitamina $\mathrm{C}$, el color superficial, el $\mathrm{pH}$, la acidez, y aumentar la concentración de carotenoides. Por otra parte, se requieren otros estudios que permitan evaluar la calidad organoléptica, estandarizar y optimizar el proceso de pasteurización y realizar estudios de estabilidad durante el almacenamiento del jugo de uchuva.

\section{REFERENCIAS}

[1] S. Kamiloglu, "Authenticity and traceability in beverages," Food Chem., vol. 277, pp. 1224, Mar. 2019. https://doi.org/10.1016/j.foodchem.2018.10.09 1.

[2] L. E. Ordóñez-Santos, J. Martínez-Girón, and M. E. Arias-Jaramillo, "Effect of ultrasound treatment on visual color, vitamin $\mathrm{C}$, total phenols, and carotenoids content in Cape gooseberry juice," Food Chem., vol. 233, pp. 96-100, Oct. 2017. https://doi.org/10.1016/j.foodchem.2017.04.11 4.

[3] J. E. Zapata M., G. L. Ciro G., and P. Marulanda L., "Optimization of pulsed vacuum osmotic dehydration of the cape gooseberry (Physalis peruviana L.) using the response surface methodology," Agron. Colomb., vol. 34, no. 2, p. 228, May 2016. https://doi.org/10.15446/agron.colomb.v34n2. 54920.

[4] K. Bravo and E. Osorio, "Characterization of polyphenol oxidase from Cape gooseberry (Physalis peruviana L.) fruit," Food Chem., vol. 197, pp. 185-190, Apr. 2016. https://doi.org/10.1016/j.foodchem.2015.10.12 6.

[5] M. F. Ramadan, "Bioactive phytochemicals, nutritional value, and functional properties of cape gooseberry (Physalis peruviana): An overview," Food Res. Int., vol. 44, no. 7, pp. 1830-1836, Aug. 2011. https://doi.org/10.1016/j.foodres.2010.12.042.

[6] L. Etzbach, A. Pfeiffer, A. Schieber, and F. Weber, "Effects of thermal pasteurization and ultrasound treatment on the peroxidase activity, carotenoid composition, and physicochemical properties of goldenberry (Physalis peruviana L.) puree," $L W T$, vol. 100, pp. 69-74, Feb. 2019. https://doi.org/10.1016/j.lwt.2018.10.032.

[7] J. Peng, J. Tang, D. M. Barrett, S. S. Sablani, N. Anderson, and J. R. Powers, "Thermal pasteurization of ready-to-eat foods and vegetables: Critical factors for process design and effects on quality," Crit. Rev. Food Sci. Nutr., vol. 57, no. 14, pp. 2970-2995, Sep. 2017. https://doi.org/10.1080/10408398.2015.10821 26.

[8] D. Cautela, D. Castaldo, and B. Laratta, "Thermal inactivation of pectin methylesterase in pineapple juice," J. Food Meas. Charact., vol. 12, no. 4, pp. 2795-2800, Dec. 2018. https://doi.org/10.1007/s11694018-9894-1.

[9] E. G. Alves Filho et al., "Evaluation of thermal and non-thermal processing effect on non-prebiotic and prebiotic acerola juices using $1 \mathrm{H}$ q NMR and GC-MS coupled to chemometrics," Food Chem., vol. 265, pp. 2331, Nov. 2018. https://doi.org/10.1016/j.foodchem.2018.05.03 8.

[10] A. Tchuenchieu et al., "Effect of low thermal pasteurization in combination with carvacrol on color, antioxidant capacity, phenolic and 
vitamin C contents of fruit juices," Food Sci. Nutr., vol. 6, no. 4, pp. 736-746, Jun. 2018. https://doi.org/10.1002/fsn3.611.

[11] M. A. Rabie, A. Z. Soliman, Z. S. Diaconeasa, and B. Constantin, "Effect of Pasteurization and Shelf Life on the Physicochemical Properties of Physalis ( Physalis peruviana L.) Juice," J. Food Process. Preserv., vol. 39, no. 6, pp. 1051-1060, Dec. 2015. https://doi.org/10.1111/jfpp.12320.

[12] Icontec Internacional, "Frutas Frescas. Uchuva. Especificaciones," Intituto Colombiano de Normas Técnicas, Bogotá, 1999.

[13] M. L. Rojas, T. S. Leite, M. Cristianini, I. D. Alvim, and P. E. D. Augusto, "Peach juice processed by the ultrasound technology: Changes in its microstructure improve its physical properties and stability," Food Res. Int., vol. 82, pp. 22-33, Apr. 2016. https://doi.org/10.1016/j.foodres.2016.01.011.

[14] C. Dhuique-Mayer, M. Tbatou, M. Carail, C. Caris-Veyrat, M. Dornier, and M. J. Amiot, "Thermal Degradation of Antioxidant Micronutrients in Citrus Juice: Kinetics and Newly Formed Compounds," J. Agric. Food Chem., vol. 55, no. 10, pp. 4209-4216, May 2007. https://doi.org/10.1021/jf0700529.

[15] V. Santhirasegaram, Z. Razali, and C. Somasundram, "Effects of thermal treatment and sonication on quality attributes of Chokanan mango (Mangifera indica L.) juice," Ultrason. Sonochem., vol. 20, no. 5, pp. 1276-1282, Sep. 2013. https://doi.org/10.1016/j.ultsonch.2013.02.005

[16] G. W. Latimer, Official Methods of Analysis of $A O A C$ International, 21st ed. Estados Unidos: The scientific Association Dedicated to Analytical Excellence, 2016.

[17] S. K. Jagota and H. M. Dani, "A new colorimetric technique for the estimation of vitamin C using Folin phenol reagent," Anal. Biochem., vol. 127, no. 1, pp. 178-182, Nov. 1982. https://doi.org/10.1016/00032697(82)90162-2.

[18] D. J. Hart and K. J. Scott, "Development and evaluation of an HPLC method for the analysis of carotenoids in foods, and the measurement of the carotenoid content of vegetables and fruits commonly consumed in the UK," Food Chem., vol. 54, no. 1, pp. 101111, Jan. 1995. https://doi.org/10.1016/03088146(95)92669-B.

[19] M.-L. Olivares-Tenorio, R. Verkerk, M. A. J. S. van Boekel, and M. Dekker, "Thermal stability of phytochemicals, HMF and antioxidant activity in cape gooseberry ( Physalis peruviana L . )," J. Funct. Foods, vol. 32, pp. 46-57, May 2017. https://doi.org/10.1016/j.jff.2017.02.021.

[20] Y. Lee and L. Howard, "Firmness and Phytochemical Losses in Pasteurized Yellow Banana Peppers ( Capsicum a nnuum ) As Affected by Calcium Chloride and Storage," J. Agric. Food Chem., vol. 47, no. 2, pp. 700703, Feb. 1999. https://doi.org/10.1021/jf980921h.

[21] E. Domínguez Romero, "Influencia de los tratamientos térmicos en la elaboración de productos untables de kiwi formulados con isomaltulosa-fructosa o sacarosa," Universidad Politécnica de Valencia, 2011.

[22] I. P. Pardo, "Influencia de las condiciones de deshidratación en los niveles de compuestos con actividad antioxidante del tomate Cherry," Universidad Politécnica de Valencia, 2007.

[23] P. Burg and P. Fraile, "Vitamin C Destruction During the Cooking of a Potato Dish," LWT - Food Sci. Technol., vol. 28, no. 5, pp. 506-514, Jan. 1995. https://doi.org/10.1006/fstl.1995.0085.

[24] P. Khandpur and P. R. Gogate, "Effect of novel ultrasound based processing on the nutrition quality of different fruit and vegetable juices," Ultrason. Sonochem., vol. 27, pp. 125-136, Nov. 2015. https://doi.org/10.1016/j.ultsonch.2015.05.008

[25] K. Aaby, I. H. Grimsbo, M. B. Hovda, and T. M. Rode, "Effect of high pressure and thermal processing on shelf life and quality of strawberry purée and juice," Food Chem., vol. 260, pp. 115-123, Sep. 2018. https://doi.org/10.1016/j.foodchem.2018.03.10 0 .

[26] A. Oliveira, M. Pintado, and D. P. F. Almeida, "Phytochemical composition and antioxidant activity of peach as affected by pasteurization and storage duration," $L W T$ Food Sci. Technol., vol. 49, no. 2, pp. 202207, Dec. 2012. https://doi.org/10.1016/j.lwt.2012.07.008.

[27] J. J. Torres Gama and C. M. Sylos, "Effect of thermal pasteurization and concentration on carotenoid composition of Brazilian Valencia orange juice," Food Chem., vol. 100, no. 4, pp. 1686-1690, Jan. 2007. https://doi.org/10.1016/j.foodchem.2005.01.06 2.

[28] L. E. Ordóñez-Santos, L. Vázquez-Odériz, E. Arbones-Maciñeira, and M. Á. RomeroRodríguez, "The influence of storage time on micronutrients in bottled tomato pulp," Food Chem., vol. 112, no. 1, pp. 146-149, Jan. 2009.

https://doi.org/10.1016/j.foodchem.2008.05.05 1.

[29] E. Reboul et al., "Enrichment of Tomato 
Cambios en los compuestos bioactivos del jugo de uchuva (Physalis peruviana L.) Pasteurizado

Paste with $6 \%$ Tomato Peel Increases Lycopene and beta-Carotene Bioavailability in Men," J. Nutr., vol. 135, no. 4, pp. 790794 , Apr. 2005. https://doi.org/10.1093/jn/135.4.790.

[30] M. L. Nguyen and S. J. Schwartz, "Lycopene: chemical and biological properties," Food Technol., vol. 53, no. 2, pp. 38-45, 1999.

[31] L. E. Ordóñez-Santos and A. VázquezRiascos, "Effect of processing and storage time on the vitamin $\mathrm{C}$ and lycopene contents of nectar of pink guava (Psidium guajava L.).," Arch. Latinoam. Nutr., vol. 60, no. 3, pp. 280-284, Sep. 2010.

[32] D. B. R. Amaya, Carotenoides y preparación de alimentos: La retención de los Carotenoides Provitamina $A$ en alimentos preparados, procesados y almacenados. Estados Unidos: Agencia para el Desarollo Internacional de los Estados Unidos, 1999. 